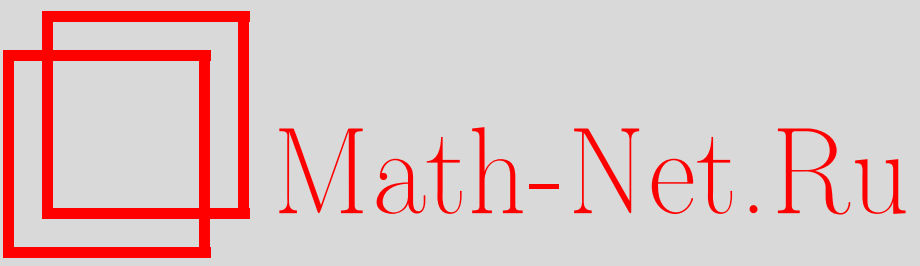

Д. А. Шакин, О функциях Гильберта и числах Бетти однородных идеалов во внешней алгебре, УМН, 2004, том 59, выпуск 5, 165-166

DOI: https://doi.org/10.4213/rm786

Использование Общероссийского математического портала Math-Net.Ru подразумевает, что вы прочитали и согласны с пользовательским соглашением

http://www.mathnet.ru/rus/agreement

Параметры загрузки:

IP: 3.80 .253 .173

26 апреля 2023 г., 12:42:01 


\title{
О ФУНКЦИЯХ ГИЛЬБЕРТА И ЧИСЛАХ БЕТТИ ОДНОРОДНЫХ ИДЕАЛОВ ВО ВНЕШНЕЙ АЛГЕБРЕ
}

\author{
Д. А. ШАКин
}

Рассмотрим внешнюю алгебру $E_{n}=k\left\langle e_{1}, \ldots, e_{n}\right\rangle$ над полем $k$ и зафиксируем в ней некоторый мономиальньй идеал $I$. Для всякого однородного идеала $J \subseteq E_{n}$ обозначим через $H_{J}$ его функцию Гильберта (т.е. $H_{J}(d)=\operatorname{dim}_{k} J_{d}$ - размерность градуированной компоненты идеала $J$ степени $d)$. В данной заметке излагаются некоторые результаты о функциях Гильберта и градуированных числах Бетти идеалов в $E_{n}$, содержащих идеал $I$.

Задача описания функций Гильберта однородных идеалов в $E_{n}($ т.е. случай $I=0)$ была рассмотрена в работах [1] и [2], где было доказано, что функция $H: \mathbb{N} \cup\{0\} \rightarrow \mathbb{N} \cup\{0\}$ является функцией Гильберта некоторого однородного идеала $J \subseteq E_{n}$ тогда и толњко тогда, когда существует такой лекссегментный идеал $L$ (см. определение ниже), что $H_{L}=H$. Для обобщения этого резултата на случай, когда $I \neq 0$, потребуются следующее два определения.

ОПредЕЛЕниЕ 1 . Идеал $L \subseteq E_{n}$ назьвается лекссегментным, если вместе с каждым мономом $u$ степени $d$ идеал $L$ содержит и все мономы степени $d$, болшшие $u$ по лексикографичекому порядку. Если зафиксирован некоторьй мономиальньй идеал $I$, то идеал $L$ назьвается $I$-лекссегментным, если его можно представить в виде $L=I+L^{\prime}$, где $L^{\prime}$ - некоторьй лекссегментный идеал.

ОПРЕДЕЛЕНИЕ 2 . Пусть $I \subseteq E_{n}$ - мономиальньй идеал. Если для всякого однородного идеала $J \subseteq E_{n}$, содержащего $I$, существует $I$-лекссегментньй идеал $L^{I}(J)$ с условием $H_{J}=H_{L^{I}(J)}$, то идеал $I$ назьвается $M$-идеалом.

Как следует из определения, если $I$ есть $M$-идеал, то все функции Гильберта однородных идеалов, содержащих $I$, исчерпываются функциями Гилберта $I$-лекссегментных идеалов. В частности, результаты работ [1] и [2] показьвают, что нулевой идеал в $E_{n}$ является $M$-идеалом. Следующие две теоремы устанавливают важные свойства $M$-идеалов:

Теорема 1. Пусть $I \subseteq E_{n}-M$-идеал. Тогда $I E_{n+1} \subseteq E_{n+1}-$ тоже $M$-идеал.

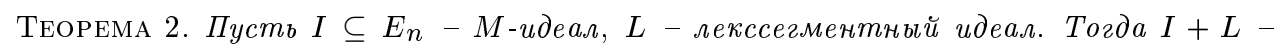
тоже $M$-идеал.

Довольно очевидно, что все лекссегментные идеалы являются $M$-идеалами. Теоремы 1 и 2 позволяют обобщить этот резултат на более широкий класс идеалов.

ОПрЕДЕлЕнИЕ 3. Идеал $I \subseteq E_{n}$ назьвается кусочно лекссегментным, если его можно представить в виде $I=L_{1} E_{n}+\cdots+L_{n} E_{n}$, где $L_{i} \subseteq E_{i}$ - некоторые лекссегментные идеалы.

Теорема 3. Всякий кусочно лекссегментный идеал $I \subseteq E_{n}$ является $M$-идеалом.

Общий ответ на вопрос, какие именно идеалы являются $M$-идеалами, по-видимому, довольно сложен; однако оказьвается возможным описать все $M$-идеалы в важном классе сильно устойчивых идеалов, которые возникают как идеалы старших членов при общей линейной замене переменных.

ОПРЕДЕлЕниЕ 4 . Мономиальньй идеал $J \subseteq E_{n}$ назьвается сильно устойчивым, если для любого монома $e_{i_{1}} \wedge \cdots \wedge e_{i_{d}} \in J, i_{1}<\cdots<i_{d}$, для любого $j \notin\left\{i_{1}, \ldots, i_{d}\right\}$ и для любого $k \in\{1, \ldots, d\}$ имеем $e_{i_{1}} \wedge \cdots \wedge e_{i_{k-1}} \wedge e_{i_{k+1}} \wedge \cdots \wedge e_{i_{d}} \wedge e_{j} \in J$.

ТЕОрема 4. Пусть $I \subseteq E_{n}-$ сильно устойчивый идеал. Идеал I является $M$-идеалом тогда и только тогда, когда он кусочно лекссегментныи.

Напомним, что всякому однородному идеалу $J \subseteq E_{n}$ можно сопоставить идеал $\operatorname{Gin}(J)$, называемьй общим идеалом старших членов, следующим образом: сначала рассматривается общая линейная замена переменных $f$ с верхнетреугольной матрицей, а потом от получившегося идеала $f(J)$ берется идеал старших членов относительно некоторого порядка на мономах (здесь мш будем рассматривать обратный лексикографический порядок); иначе говоря, $\operatorname{Gin}(J)=\operatorname{In}(f(J))$, где In - операция взятия идеала старших членов. Тот факт, что все кусочно лекссегментные идеалы оказываются сильно устойчивыми, позволяет сформулировать следующую теорему.

Работа выполнена при частичной финансовой поддержке Российского фонда фундаментальных исследований (грант № 02-01-00468). 


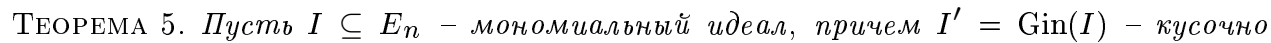
лекссегментный. Если функция $H: \mathbb{N} \cup\{0\} \rightarrow \mathbb{N} \cup\{0\}$ является функцией Гильберта некоторого однородного идеала $J$, содержащего I, то существует такой $I^{\prime}$-лекссегментный идеал $L$, что $H_{L}=H$.

Известно (см., например, [3], [4]), что лекссегментные идеалы в алгебре $E_{n}$ имеют максимальные градуированные числа Бетти среди всех однородных идеалов с фиксированной функцией Гильберта. Как показьвается следующая теорема, это справедливо и для $I$-лекссегментных идеалов.

TeOpema 6. Пусть $\operatorname{char} k=0$. Пусть также $I \subseteq E_{n}-$ кусочно лекссегментный идеал, а $J$ - однородньй идеал, содержащий $I$. Тогда для всех $i, j \geqslant 0$ выполнень неравенства

$$
\beta_{i j}^{E_{n}}(J) \leqslant \beta_{i j}^{E_{n}}\left(L^{I}(J)\right), \quad \beta_{i j}^{A_{n}}(S(J)) \leqslant \beta_{i j}^{A_{n}}\left(S\left(L^{I}(J)\right)\right),
$$

где $A_{n}=k\left[x_{1}, \ldots, x_{n}\right]$ - кольцо коммутативных многочленов над $k$, a $S(J)$ - однородный идеал в $A_{n}$, порожденный такими бесквадратными многочленами $f\left(x_{1}, \ldots, x_{n}\right) \in$ $A_{n}$, ито $f\left(e_{1}, \ldots, e_{n}\right) \in J$.

Пусть $\delta(I)$ - максимальная из степеней порождающих идеала $I$. Несложно заметить, что если $I-M$-идеал, а $V \subseteq\left(E_{n}\right)_{d}$ - однородное векторное пространство, причем $d \geqslant \delta(I)$ и $I_{d} \subseteq V$, то вьполнено следующее неравенство:

$$
\operatorname{dim}_{k} \mathrm{e} V \geqslant \operatorname{dim}_{k} \mathrm{e} L^{I}(V),
$$

где $L^{I}(V)=\left(L^{I}\left(V E_{n}\right)\right)_{d}$, а $\mathrm{e} V \subseteq\left(E_{n}\right)_{d+1}$ - векторное пространство, порожденное произведениями вида $f \wedge e_{i}$, где $f \in V$. Особый интерес представляют пространства, для которых неравенство (1) превращается в равенство.

ОПРЕДЕЛЕНИЕ 5 . Пространство $V \subseteq\left(E_{n}\right)_{d}$ назьвается $I$-гоцмановым, если выполнены следующие условия: $d \geqslant \delta(I), I_{d} \subseteq V$ и $\operatorname{dim}_{k} \mathrm{e} V=\operatorname{dim}_{k} \mathrm{e} L^{I}(V)$. Идеал $J \subseteq E_{n}$ называется $I$-гоцмановым, если $J_{d}=I_{d}$ при $d<\delta(I)$ и $J_{d}$ является $I$-гоцмановым при $d \geqslant \delta(I)$.

Справедлива следующая теорема, обобщающая результаты работ [5] и [3].

Теорема 7. Пусть $I \subseteq E_{n}-$ кусочно лекссегментный идеал, $a V \subseteq\left(E_{n}\right)_{d}-I$-гоцманово векторное пространство, тогда $\mathrm{e} V$ - тоже $I$-гоцманово.

Из определения $I$-гоцманова идеала следует, что нулевые градуированные числа Бетти $I$-гоцманова идеала $J$ и соответствующего ему $I$-лекссегментного идеала $L^{I}(J)$ совпадают. Как показывает следующая теорема, это справедливо и для старших чисел Бетти.

ТеОрема 8. Пусть $\operatorname{char} k=0$. Пусть также $I \subseteq E_{n}-$ кусочно лекссегментный идеал, а $J-I$-гоцманов идеал. Тогда для всех $i, j \geqslant 0$ выполнено равенство

$$
\beta_{i j}^{E_{n}}(J)=\beta_{i j}^{E_{n}}\left(L^{I}(J)\right) \text {. }
$$

Если, в дополнение, идеал $J$ является мономиальным, то

$$
\beta_{i j}^{A_{n}}(S(J))=\beta_{i j}^{A_{n}}\left(S\left(L^{I}(J)\right)\right) .
$$

\section{СПИСОК ЛИТЕРАТУРЫ}

[1] J. Kruskal // Mathematical Optimization Techniques / ed. R. Bellman. Berkley: Univ. of California Press, 1963. P. 251-278. [2] G. Katona // Theory of Graphs / ed. P. Erdös, G. Katona. New York: Academic Press, 1968. P. 187-207. [3] A. Aramova, J. Herzog, T. Hibi // J. Algebra. 1997. V. 191. № 1. P. 174-211. [4] A. Aramova, J. Herzog, T. Hibi // J. Algebraic Combin. 2000. V. 12. № 3. P. 207-222. [5] G. Gotzmann // Math. Z. 1978. V. 158. № 1. P. 61-70.

Московский государственньй

Принято редколлегией университет им. М.В. Ломоносова 23.07.2004

E-mail: dshakin@online.ru 